\title{
PRÁCTICAS PARA MEJORAR LA REGULACIÓN EN COLOMBIA
}

Mario Andrés Pinzón Camargo*

$\mathrm{E}^{1}$ 1 papel del Estado en la sociedad ha fluctuado entre la intervención y la no intervención conforme a las funciones que se le han asignado. Así, las funciones del Estado liberal imperante en el siglo XIX eran asegurar "la vida, la propiedad y la libertad mediante la protección contra el fraude, el hurto, el incumplimiento contractual o los daños ocasionados por terceros"(Cepeda, 2003). Es decir, su papel se limitaba al de un Estado gendarme. Ante el fracaso de este tipo de Estado durante la Gran Depresión, tuvo que cambiar e intervenir directamente en los asuntos sociales, sobre todo de orden económico. Esta nueva función llevó a la aparición de un Estado empresario que refrenaba y orientaba la iniciativa privada para evitar comportamientos contrarios al bienestar social. En los años setenta, durante la crisis del petróleo, cobró fuerza la idea de reducir la participación del Estado en la economía para evitar las fallas del gobierno, como las llamó la escuela de la elección pública ${ }^{1}$.

Hoy, después de las crisis de la deuda de los ochenta, de la crisis asiática de los noventa, de la crisis de las empresas de alta tecnología y de la crisis inmobiliaria en Estados Unidos, se acepta que el Estado debe intervenir para prever, supervisar y corregir el comportamiento

* Magíster en Derecho Económico, asesor del Ministerio de Comercio, Industria y Turismo de Colombia, docente de la Universidad Externado de Colombia, Bogotá, Colombia, [pinzonmario@hotmail.com]. Fecha de recepción: 16-07-2014, fecha de modificación: 11-03-2015, fecha de aceptación: 20-10-2016. Sugerencia de citación: Pinzón C, M. A. "Prácticas para mejorar la regulación en Colombia", Revista de Economia Institucional 18, 35, 2016, pp. 207-228. DoI: http://dx.doi. org/10.18601/01245996.v18n35.11.

${ }^{1}$ Debidas a cuatro causas que explican la incapacidad sistemática para cumplir sus objetivos: "su reducida información, su reducido conocimiento de la respuesta privada a su intervención, su reducido control de la burocracia y las limitaciones que impone el proceso político" (Stiglitz, 2000, 16-17). 
socialmente indeseable de los agentes. De modo que vivimos el paso de un Estado gestor, que proporcionaba bienes y servicios, a un Estado regulador que deja al sector privado actividades que antes estaban a su cargo. Esta situación suscita un desafío para el Estado desde el punto de vista de las técnicas necesarias para orientar el comportamiento de los agentes privados en espacios de tradicional dominio estatal (Muñoz, 2011, 209).

En este contexto, y ante las nuevas funciones que debe cumplir el Estado, es importante crear marcos regulatorios e institucionales que fomenten la actividad económica y contribuyan a lograr fines superiores como el bienestar social. A la luz de algunos conceptos de la nueva economía institucional (NEI), este artículo analiza la relación entre las prácticas para mejorar la regulación y la legitimidad del Estado. En la primera sección se presenta el marco conceptual. La segunda analiza el concepto de regulación y expone las motivaciones que le dan origen. La tercera revisa las características del proceso regulatorio en Colombia y su relación con la legitimidad del Estado. En la cuarta se presenta un estudio de caso y las prácticas adoptadas para mejorar la calidad de la regulación. Por último se presentan algunas conclusiones.

\section{LA REGULACIÓN Y LA NUEVA ECONOMÍA INSTITUCIONAL}

En la nueva economía institucional concurren enfoques históricos, sociológicos y económicos basados en la elección racional (Roth, 2002; Baldwin et al., 2012). En este escrito se adopta el enfoque económico, del cual se pueden distinguir al menos cuatro vertientes: neoclásica, contractualista, evolucionista e histórica (Ayala, 2005, 56-71). Uno de sus conceptos clave es el de instituciones, que se interpreta de diversas maneras ${ }^{2}$, y que North define como "las reglas de juego de una sociedad o, más formalmente, las limitaciones ideadas por el hombre que dan forma a la interacción humana" $(2006,14)$. Estas se pueden dividir en instituciones informales y formales: "limitaciones formales (reglas, leyes y constituciones) y limitaciones informales (normas de comportamiento, convenciones y códigos de conducta auto impuestos)" (North, 1993).

\footnotetext{
${ }^{2}$ Para Ayala "son el conjunto de reglas que articulan y organizan las interacciones económicas sociales y políticas entre los individuos y los grupos sociales" (ibíd., 63). Según Nelson y Sampat, son "las maneras estándar y esperadas de interacción de los agentes económicos, para lograr determinados resultados" (2001, 30). Y según Arias, son "soluciones relativamente eficientes a los problemas de acción colectiva [...] para reducir los costos de transacción entre individuos y grupos, y aumentar la eficiencia" $(2008,51)$.
} 
Una función de las instituciones es reducir los costos de transacción (Williamson, 2009, 27; San Emeterio, 2006, 68), dado que el uso del sistema de precios tiene un costo (Coase, 1937,390) y es irreal pensar en transacciones que no tengan ese costo (Coase, 1960, 15). "Los costos de transacción son el equivalente económico de la fricción en los sistemas físicos" (Williamson, 2009, 29), e incluyen los costos de búsqueda, negociación y vigilancia descritos por Coase $(1960,15)$. De acuerdo con Williamson, estos se pueden clasificar a su vez en costos ex ante y ex post (2009, 30-32). Esta clasificación sirve para reinterpretar el teorema de Coase en lo que se denomina teorema normativo de Hobbes y teorema normativo de Coase. La primera reinterpretación sugiere que la ley se debe formular "de modo que elimine los impedimentos para los acuerdos privados"; y, la segunda, "de modo que minimice el daño causado por las fallas de los acuerdos privados" (Cooter y Ulen, 1997, 128 y 129).

En suma, las normas o leyes, entendidas como instituciones formales, disminuyen los costos de transacción y garantizan que los bienes $\mathrm{y}$ servicios queden a cargo de quienes los provean mejor. $\mathrm{Y}$ puesto que en el mercado hay fricciones, que se reflejan en dichos costos, se requiere que el Estado intervenga para eliminar las barreras que impiden o limitan las transacciones. Uno de sus instrumentos es la regulación, tema que trata la siguiente sección.

\section{REGULACIÓN: CONCEPTO Y MOTIVACIONES}

Para hablar de una mejor regulación es necesario definir en qué consiste ${ }^{3}$. Es difícil definirla porque varía según el contexto (Ponce, 2003) y porque se puede interpretar en distintos sentidos, como un conjunto de comandos, como una influencia estatal deliberada o como toda forma de influencia social y económica (Baldwin et al., 2012). Aquí se toma como guía a Muñoz $(2011,206)$ y a Baldwin et al., que la definen en sentido amplio como una actividad que refleja la capacidad de intervención del Estado en la economía y en la sociedad. Esta actividad se concreta en restricciones o habilitaciones que incentivan el comportamiento de los agentes públicos y privados, en búsqueda del interés general. A la luz de los conceptos presentados en la sección anterior, la regulación es un resultado del diseño y la implementación de instituciones formales cuyo propósito es reducir los costos de transacción para desincentivar o motivar comportamientos que puedan perjudicar o favorecer el interés general.

\footnotetext{
${ }_{3}$ Existen muy diversas definiciones; ver, por ejemplo, Boyer (1989), Ramírez (2001), Ponce (2003), Custos (2009), Grau (2009) y Baldwin et al. (2012).
} 
Además de definir la regulación es necesario considerar sus motivaciones. La literatura presenta tres explicaciones que no son excluyentes. La primera, quizá la más conocida, es que "las compañías pueden no tomar en cuenta todo el costo social de sus actividades sin intervención del gobierno" (Guasch y Spiller, 1999, 3). Esta es la explicación clásica de la intervención del Estado, mediante la regulación de la economía, para corregir fallas de mercado. La segunda, expuesta por Cuevas (2002), Barcelona (2010) y Baldwin et al. (2012), señala que el buen funcionamiento del mercado puede llevar a fallas de equidad que justifican la intervención del Estado.

En los años noventa se reconoció una tercera motivación, no asociada a las fallas sino a las ventajas de un mejor esquema institucional para desarrollar actividades económicas y sociales. Esta motivación se basaba en la idea de generar condiciones más favorables para competir en un mundo globalizado: "racionalizar la economía, promover la productividad y la competitividad" (Correa, 2009, 182). La idea de competencia desbordó las fronteras del Estado-nación y se trasladó al mercado global, donde un marco regulatorio más eficiente generaría ambientes atractivos para la inversión extranjera y la actividad productiva.

Esta concepción inspiró estudios como el Doing business auspiciado por el Banco Mundial. Desde 2002, este informe determina qué países tienen esquemas regulatorios que favorecen la actividad empresarial y cuáles la limitan. Uno de sus criterios básicos es que

la actividad económica requiere buenas normas. Entre estas se incluyen las que establecen y esclarecen los derechos de propiedad, las que reducen el costo de resolución de disputas, las que permiten que las interacciones económicas sean más predecibles y las que proporcionan a las partes contratantes importantes medidas de protección contra los abusos. El objetivo: regulaciones diseñadas para ser eficientes, accesibles a todo el que necesite recurrir a ellas y de sencilla aplicación (Banco Mundial, 2011, 16).

El cuadro 1 muestra la posición de Colombia desde 2007. En 2015 se consideró que era el país más reformador de América Latina y ascendió al puesto 34, debido a la adopción de la Ley de Garantías Mobiliarias, que favoreció el acceso a crédito empresarial. La gráfica 1 muestra su buen desempeño en comparación con el de otros países de la región, el cual puede indicar al mercado que existe un entorno más competitivo, la tercera motivación de la regulación. 
Cuadro 1

Colombia en el escalafón Doing business

\begin{tabular}{ccccccccc}
\hline 2007 & 2008 & 2009 & 2010 & 2011 & 2012 & 2013 & 2014 & 2015 \\
\hline 79 & 66 & 49 & 38 & 47 & 42 & 45 & 43 & 34
\end{tabular}

\begin{tabular}{lllllllll} 
de 175 & de 178 & de 181 & de 183 & de 183 & de 182 & de 185 & de 189 & de 189 \\
\hline
\end{tabular}

La posición 1 es la mejor.

Fuente: Banco Mundial (2014), elaboración propia.

\section{Gráfica 1}

Escalafón Doing business 2014-2015, LAc7

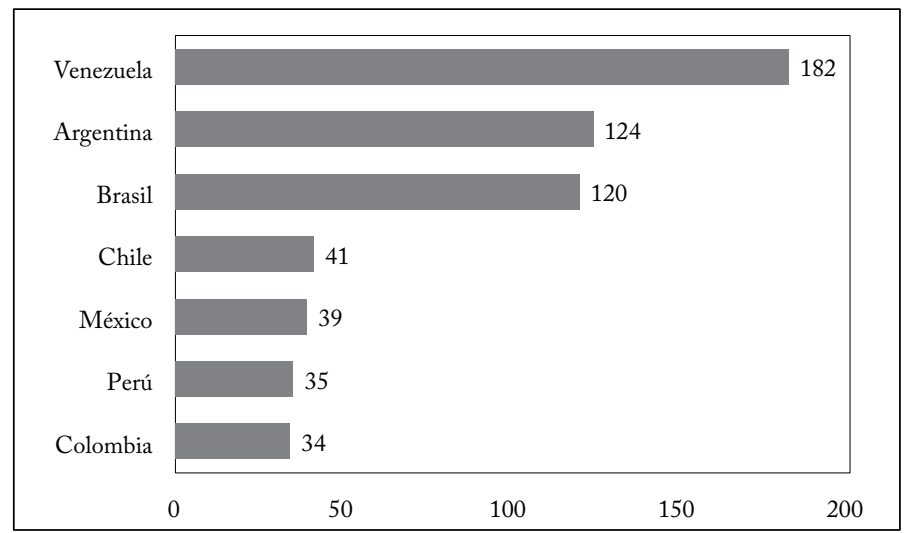

Fuente: Banco Mundial (2014), elaboración propia.

Gráfica 2

Calidad regulatoria, 2013

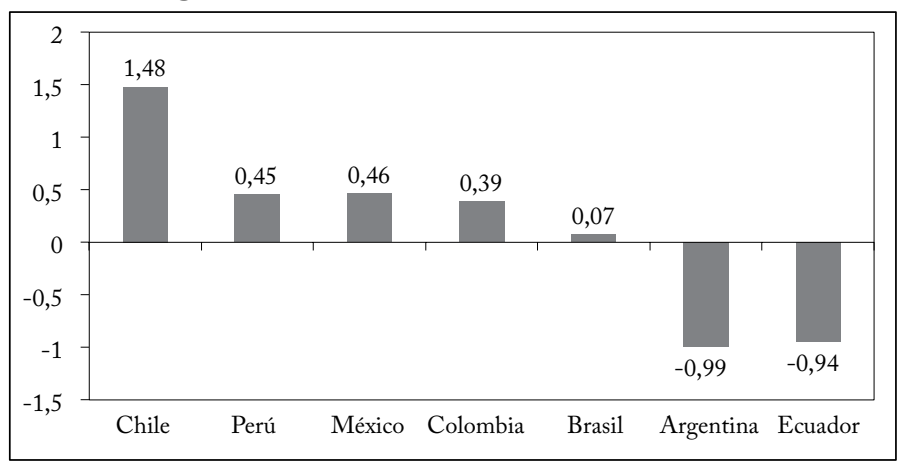

La mejor puntuación es 2,5 y la peor es $-2,5$.

Fuente: Indicadores mundiales de gobernabilidad, wGi (Banco Mundial, 2014), elaboración propia.

Otro trabajo interesante del Banco Mundial es el Worldwide Governance Indicators (WGI), que busca medir la gobernabilidad analizando 
seis variables ${ }^{4}$, entre las que se destacan la calidad regulatoria, que capta las "percepciones de la habilidad del gobierno para formular e implementar políticas y regulaciones que permitan y promuevan el desarrollo del sector privado", y el Estado de derecho, que capta "las percepciones del grado en que los agentes confían y respetan las reglas de la sociedad, en particular la calidad del cumplimiento de los contratos, los derechos de propiedad, la vigilancia y las cortes, así como la probabilidad de crímenes y violencia” (World Bank, 2011). Como se observa en la gráfica 2, en América Latina, Chile registra el mejor desempeño, mientras que Argentina obtiene la peor calificación. E1 desempeño de Colombia es superior al promedio de los países que se incluyen en la gráfica.

El centro de investigación del HsвC adopta un enfoque similar para proyectar el desarrollo económico considerando la gobernabilidad económica, el capital humano y el nivel inicial de ingreso per cápita; donde la primera categoría agrupa los componentes de estabilidad monetaria, derechos políticos y nivel de democracia, Estado de derecho y tamaño del gobierno (HSBC, 2011). En este escrito se resalta el "Estado de derecho", que "mide el atractivo del clima de inversión con base en el nivel de cumplimiento de la ley, de la sacralidad de los contratos y de los derechos de propiedad" (ibíd., 15). Los aspectos contemplados tienen una relación estrecha con la calidad del sistema jurídico, que facilita el desarrollo de actividades productivas.

Por su parte, el Foro Económico Mundial publica Global economic competitiveness, un reporte similar al Doing business, que evalúa 140 países para determinar su competitividad. Uno de sus doce pilares es el ambiente institucional, determinado por el marco legal y administrativo en el que interactúan individuos, firmas y gobiernos para generar riqueza (World Economic Forum, 2011, 4). Las gráficas 3 a 5 muestran algunos de los indicadores que se asocian a ese pilar. La gráfica 3 muestra la posición de algunos países latinoamericanos y los promedios de la región y de la OCDE. En el eje izquierdo se representa la puntuación obtenida al preguntar qué tan fácil es obtener información de los cambios en las políticas del gobierno y las regulaciones que afectan las actividades de los empresarios. El promedio de América Latina es un punto inferior al de la ocDe y se sitúa en la mitad de la escala. Esto indica que no es fácil acceder a la información necesaria para advertir los cambios regulatorios, y sugiere que

${ }^{4}$ Voz y rendición de cuentas, estabilidad política y ausencia de violencia, efectividad del gobierno, calidad regulatoria, Estado de derecho y control de la corrupción. 
hay mayores costos de transacción para conocer las normas vigentes $\mathrm{y}$ sus modificaciones.

La gráfica 4 indica el lugar de algunos países de América Latina entre 144 países en cuanto a la eficiencia de los mecanismos de solución de controversias. Esta calificación se deriva de preguntar qué tan eficiente es la infraestructura legal para la solución de controversias en el sector privado. Conforme a los conceptos expuestos en la sección anterior este indicador mide la capacidad de las instituciones formales para disminuir los costos de transacción. En otras palabras, evalúa el cumplimiento del teorema de Hobbes, pues indica qué tan eficientes son las instituciones formales para eliminar los defectos de los acuerdos privados. En los extremos de la gráfica figuran Venezuela, con el peor sistema institucional para solucionar controversias, y Chile, con el mejor sistema de la región.

Gráfica 3

Transparencia en la elaboración de política pública

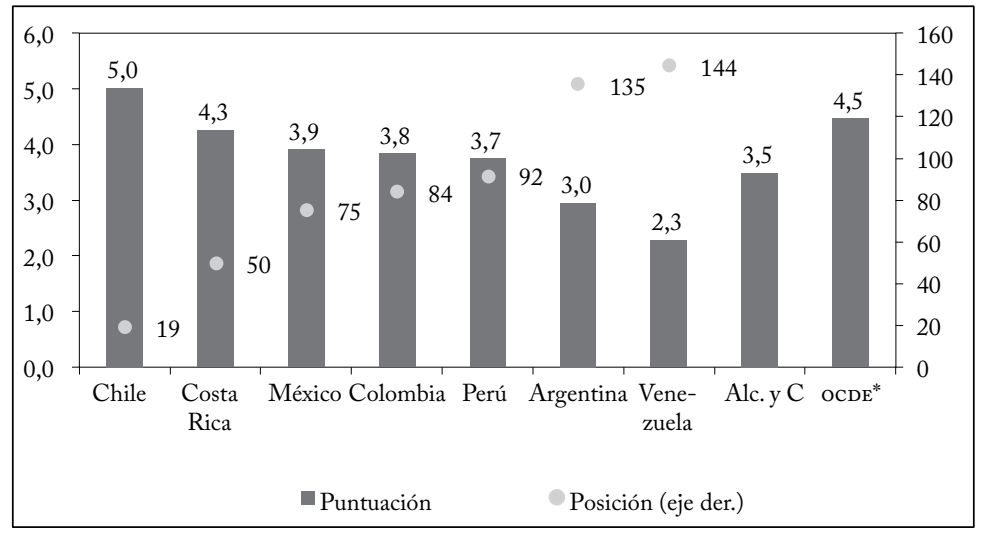

* Promedio simple de los países de la ocDE. La puntuación varía de 1 a 7 , donde 7 es muy fácil; la posición 1 es la mejor posible.

Fuente: Foro Económico Mundial (2014), encuesta de opinión en 144 países.

La gráfica 5 representa la carga (o costos de transacción) por el cumplimiento de la regulación impuesta por el gobierno. Esta gráfica indica la carga de las empresas para cumplir los requerimientos administrativos del gobierno. América Latina tiene una calificación de 3, bastante cercana al promedio de los países de la OCDE. 


\section{Gráfica 4}

Eficiencia de los mecanismos legales para la solución de controversias

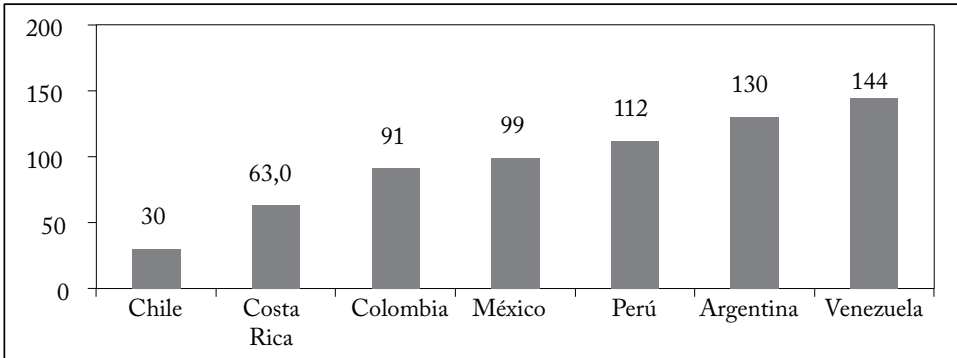

* Promedio simple de los países de la ocde. La posición 1 es la mejor posible.

Fuente: Foro Económico Mundial (2014), encuesta de opinión en 144 países.

\section{Gráfica 5}

Carga de la regulación del gobierno

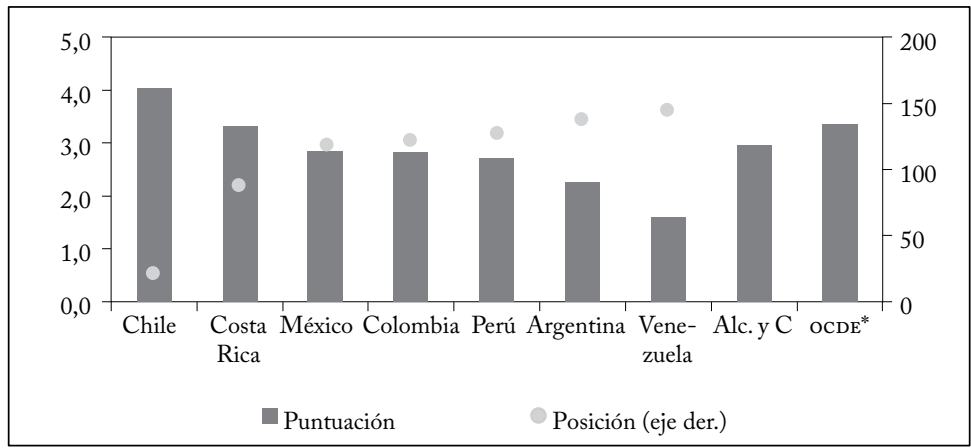

* Promedio simple de los países de la ocDE. La puntuación varía de 1 a 7 , donde 7 indica que la carga no es un inconveniente para hacer negocios: 1 es la mejor posible.

Fuente: Foro Económico Mundial (2014), encuesta de opinión en 144 países.

En suma, existen numerosos estudios y proyectos que analizan y cuantifican la calidad, la eficacia y la eficiencia de los marcos regulatorios considerando el Estado de derecho o la calidad de la regulación ${ }^{5}$, como resultado de

la emergencia de un Estado regulador y la consolidación de un nuevo movimiento de gerencia pública, que ha provocado la atención de académicos, organizaciones internacionales y gobiernos respecto de la extensión y calidad de conceptos y medidas de la regulación. De hecho, indicadores de calidad de la regulación, medidas de desempeño de la gobernabilidad regulatoria y metas para la reducción de trámites administrativos y papeleos han venido a ser puntos relevantes en la agenda de reforma de gobiernos y organizaciones internacionales (Radaelli y de Francesco, 2010, 1).

${ }^{5}$ Para más detalles, ver Radaelli y de Francesco (2010), que agrupa y analiza varios estudios y los indicadores más utilizados para evaluar la calidad regulatoria. 
Una mejor regulación es de gran importancia porque hace posible emplear mejores herramientas para corregir fallas del mercado o problemas de equidad y ayuda a crear las condiciones necesarias para mejorar la competitividad, entendida como un medio para aumentar el bienestar de la sociedad y de los individuos, una de las finalidades del Estado social de derecho.

A la luz de la nueva economía institucional, la búsqueda de una mayor competitividad requiere instituciones que reduzcan los costos de transacción. Por ello es conveniente examinar y comparar las prácticas de diferentes países. La métrica que emplean los estudios antes mencionados facilita la comparación de los marcos institucionales exitosos, aunque se debe evitar la tentación de imitarlos o trasplantarlos ${ }^{6}$.

\title{
LA REGULACIÓN COMO ACTIVIDAD ESTATAL Y LEGITIMIDAD
}

Luego de considerar qué es la regulación e identificar sus motivaciones, en esta sección se analizan los aspectos jurídicos que la caracterizan, haciendo referencia al caso colombiano. En principio, para que una actividad del Estado tenga carácter estatal

\begin{abstract}
ha de conectarse con una norma jurídica que así lo establezca, norma jurídica que en esta medida se nos of rece como una regla de atribución [...] En este sentido, toda acción administrativa ha de estar condicionada por la existencia de una norma previa que admita semejante acción, pues en caso contrario podría ser considerada como acción de la administración, imputable a la misma (Carro y Gómez, 1978, 945).
\end{abstract}

Carro y Gómez (1978) e Ibáñez (2003) plantean que en la actividad reguladora del Estado se pueden distinguir dos niveles. E1 primero corresponde a los temas que por mandato constitucional se deben reglamentar a través de una ley orgánica, la cual "se caracteriza por los requisitos especiales que exige su aprobación, modificación o derogación" (Carro y Gómez, 1978, 966), y el segundo a las materias reservadas a la ley ordinaria. De acuerdo con Ibáñez, hay situaciones donde la ley regula una materia dando espacio a la reglamentación posterior, y otras donde la ley reglamenta un mandato constitucional que en sí mismo constituye un acto de regulación. Cabe añadir que también se pueden realizar actos de regulación mediante convenios o tratados internacionales (Carro y Gómez, 1978, 983), que en el caso colombiano hacen posible, a través del bloque de constitucionalidad, la aparición de deberes u obligaciones que lleven a cambiar las reglas de juego en algunos campos.

\footnotetext{
${ }^{6}$ Aunque no se mencionaron detalle, no se pueden perder de vista las instituciones informales. Chang (2006) advierte que no se deben trasplantar instituciones formales sin una adecuada adaptación al entorno informal del país receptor.
} 
Correa (2009), quien también percibe dos niveles de ejercicio de la actividad reguladora, plantea que la intervención se puede llevar a cabo mediante normas generales y normas específicas. Las normas generales establecen disposiciones abiertas que "habilitan al Estado para ordenar u orientar la economía en general". Las normas específicas atribuyen competencias puntuales a las "autoridades públicas para imponer reglas de dirección económica o con impacto directo sobre su funcionamiento" 7 . Su especificidad depende del "área de la economía sobre la cual actúan" (ibíd., 180).

En suma, la regulación es una manifestación de la potestad de intervención del Estado en las esferas económica y social, que implica necesariamente una habilitación legal previa para ejercerla, la cual hace posible la creación de normas generales y específicas.

Los elementos que se han presentado permiten analizar las implicaciones de una mejor regulación mediante el sistema jurídico. E1 elemento articulador es la legitimidad. "El concepto de legitimidad implica que los medios para lograr los objetivos de la organización, así como los propios objetivos, estén en armonía con ciertos objetivos y necesidades aceptados por la sociedad" (Laufer y Burlaud, 1989, 28).

Nuestro análisis se refiere a la legitimidad legal de la administración pública. Para Prats, esta hoy se asocia no solo a valores como la equidad y la participación social, sino a la eficacia y la eficiencia en el uso y administración de los recursos públicos. Una visión consistente con la motivación de promover ambientes institucionales más competitivos.

a diferencia de lo que ha sucedido con las "modernas técnicas de gestión" de la reforma administrativa de los años cincuenta y sesenta, el management no se presenta ahora como un mero añadido tecnocrático al orden tradicional de la legitimidad legal de las administraciones, sino como portador de un propio factor de legitimidad, representado por los valores de eficacia y eficiencia, ya constitucionalmente reconocidos (Prats, 1996, 183).

Laufer y Burlaud (1989) hacen un recuento histórico de la relación entre legitimidad y administración. Sus principales resultados son también consistentes con los cambios en la función del Estado mencionados al comienzo del artículo. En el Estado gendarme, la legitimidad se asociaba a la elección popular; en el Estado de bienestar, a los resultados de sus acciones para corregir las fallas del mercado;

${ }^{7}$ Las normas específicas se pueden clasificar en tres grupos. E1 primero está integrado por directivas, direcciones y planes, que buscan orientar la actividad privada para lograr el interés público. El segundo, por disposiciones que si bien no prohíben la acción de los agentes, la condicionan al cumplimiento de ciertos requisitos. E1 tercero, por instrucciones que "afianzan y consolidan el funcionamiento ordenado del mercado” (ibíd., 181). 
con el advenimiento del Estado regulador, la legitimidad no solo se asocia a los resultados sino, además, a los medios que utiliza.

Por ello, la calidad de la regulación y su mejoramiento son esenciales para la legitimidad legal y la legitimidad de la administración. Unas reglas de juego más claras no solo atraen inversionistas y mejoran la capacidad productiva, sino que aumentan la credibilidad del Estado y de su capacidad de gestión. Una mejor regulación permite construir marcos institucionales que contribuyen a cumplir sus objetivos, usar de manera eficiente los recursos necesarios para su construcción y su funcionamiento, $\mathrm{y}$ reducir los costos de transacción ex ante y ex post. Pero dada la necesidad de considerar los fines y los medios, no basta que la ley sea eficaz para cumplir esos objetivos, también debe ser eficiente.

\section{LA EFICIENCIA DE LA REGULACIÓN EN COLOMBIA}

La mejora de la regulación no solo promueve un ambiente más competitivo sino que ayuda a legitimar el Estado. Según Ponce (2011), la crisis actual ha llevado a que el examen de dicha legitimidad no se límite a evaluar qué y cuánto; además, se debe considerar cómo se lleva a cabo los procesos, lo que hace posible emplear conceptos como la eficiencia y la eficacia para evaluar la legitimidad por resultados teniendo en cuenta los recursos empleados para alcanzarlos.

Esta preocupación por los medios ha llevado, por ejemplo, a adoptar, en el caso europeo, el principio de la buena administración como un derecho fundamental. Principio que configura una arena propicia para discutir la pertinencia de ideas como la eficiencia y la eficacia económica en la actuación del Estado. Con esto último se esperaría que el uso de técnicas para medir el impacto económico de las decisiones administrativas fuese una actividad necesaria y cotidiana (ibíd., 461).

Consideraciones de este tipo no solo se presentan en la Unión Europea. Ariño y de la Cuétara señalan que, en Estados Unidos, "la aplicación del examen 'costo-beneficio' a un centenar de regulaciones existentes dio como resultado que la mitad de ellas costaban más de lo que aportaban" $(2000,26)$. Ponce explica que en este mismo país:

Del tradicional control laxo sobre el modo de ejercicio de la discrecionalidad administrativa se pasó progresivamente a un escrutinio riguroso de la corrección de su desarrollo, basado en el análisis conjunto del expediente y de la motivación de la decisión normativa, a fin de comprobar que la alternativa elegida había sido el fruto de una ponderación auténtica de los intereses sociales, a los que tenía que haber escuchado realmente en los trámites participativos existentes $(2003,100)$. 
Con este panorama, es evidente la necesidad de que el proceso de construcción de las normas por medio del cual, como se explicó atrás, se habilita la actuación del Estado atienda a una serie de consideraciones de eficiencia y eficacia. En este orden es fundamental que "[1] as medidas regulatorias deben basarse en el análisis económico de las decisiones, que permita prever las consecuencias de las mismas, no solo a corto plazo, sino también a medio y largo plazo" (Ariño y de la Cuétara, 2000, 11); en el desarrollo del instrumento jurídico así como en su implementación y cumplimiento.

Tradicionalmente, el análisis económico que proponen Ariño y de la Cuétara (2000) se ha predicado de aquellas acciones y decisiones administrativas con cargo a recursos públicos o que afectan el desarrollo de las actividades productivas. En el campo internacional, las consideraciones expresadas hasta este punto se han consolidado en una corriente conocida como política de mejora regulatoria.

Esta política es promovida como una buena práctica de los países de la OCDE. De acuerdo con esta organización, la mejora de la regulación, junto con las políticas fiscal y monetaria, es una de las tres palancas de que dispone el gobierno para manejar la economía, ejecutar políticas e influir en el comportamiento. Esta tesis se apoya en estudios como los de Jacobzone et al. (2010) y Jalilian et al. (2007), que sugieren que una mejor regulación impacta positivamente el crecimiento del PIB de un país. Así mismo, la investigación adelantada por Gorgens et al. (2003) demuestra que una economía sobre regulada puede tener un crecimiento económico entre un $2 \%$ y un $3 \%$ menor que una menos regulada. El trabajo de Djankov et al. (2006) concluye que, para un país, pasar del peor cuartil al mejor, implica un incremento anual promedio del 2,3\% en su crecimiento.

En el campo operativo, la política de mejora normativa busca "asegurar que las regulaciones y los marcos regulatorios están justificados, tienen alta calidad y buscan objetivos de política pública. Esta política ayuda a los hacedores de política a tomar decisiones informadas acerca de qué regular, a quién regular y cómo regular" (OECD, 2011, 7). Como instrumentos básicos de esta política se contempla el análisis de impacto regulatorio (RIA) ${ }^{8}$, la consideración de alternativas a la regulación, la simplificación administrativa, la transparencia regulatoria mediante la consulta pública y la evaluación ex post de la regulación (oECD, 2011, 8).

\footnotetext{
${ }^{8}$ Este instrumento fue introducido en Colombia, con el nombre de análisis de impacto normativo (AIN), mediante el documento CONPES 3816 de 2014.
} 
A la luz de la experiencia colombiana este tipo de prácticas resultan bastante recientes. La política anti-trámites consignada en los planes de desarrollo 2002-2006, 2010-2014 y 2014-2018 ha buscado disminuir costos de transacción que afectan tanto al ciudadano como al empresario en su quehacer diario. La primera etapa de este proceso condujo a la expedición del Decreto Ley 19 de 2012 -más conocido como Ley anti-trámites-, el cual supuso un primer hito en el proceso de simplificación regulatoria del país.

En esta misma línea se destaca el Decreto 1345 de 2010, conocido como el Decreto de decretos, que establece una guía para la expedición de estos instrumentos regulatorios. En esta norma se contempla la necesidad de incluir procesos de consulta pública más claros, llevar a cabo un análisis costo-beneficio ex ante, y emplear lenguaje claro en su redacción. No obstante, debido a la institucionalidad informal del país es evidente la falta observancia de estas técnicas de producción normativa. De hecho, como lo sugiere el estudio de la ocDe sobre la política regulatoria en Colombia:

E1 Decreto 1345 de 2010 establece guías para publicar regulaciones y, en su artículo 11, pone de manifiesto que "las propuestas deben caracterizarse por su claridad, precisión, sencillez y coherencia, en forma tal que no exista ambigüedad ni contradicciones". Sin embargo, ninguna institución supervisa la implementación del decreto, y el uso apropiado de las guías depende del criterio de quienes redactan las leyes. Por consiguiente, se cuenta con escasa evidencia del uso de estas guías y del impacto que puedan haber ejercido en la calidad de la regulación (OCDE, 2014).

Otro indicador del proceso regulatorio en Colombia es la proliferación de normas, la llamada inflación normativa. Según el documento conpes 3816 de 2014, "durante los años de 2007 y 2011, el Poder Ejecutivo expidió 4.039 decretos, de los cuales 1.645 correspondieron a decretos sustanciales y los demás a actos administrativos de trámite, de nombramiento y otros de carácter particular" (DNP, 2014). Es decir, en ese periodo se expidieron 807 decretos por año, unos 2,5 por día. Y en cuanto a la expedición de leyes, entre 1992 y 2011 se expidieron en promedio 75,2 cada año (Pinzón, 2013, 216).

Sumado a las dificultades advertidas en el Decreto de decretos, este deja discreción a quienes elaboran estos cuerpos institucionales para que definan si se debe considerar o no la relación costo-beneficio. Esto se evidencia en el artículo 5, el cual tiene como finalidad definir el contenido de la memoria justificativa de los decretos y resoluciones. En el numeral 4 de dicho artículo se establece:

Impacto económico si fuere el caso, el cual deberá señalar el costo o ahorro, de la implementación del respectivo acto (2010). 
Hasta este punto solo se han señalado dos componentes que permiten evidenciar el desarrollo de una política de mejora regulatoria o normativa ${ }^{9}$ en el país. Adicional a estas, como lo señala estudio de la ocDE sobre la política regulatoria en Colombia, se han implementado otras prácticas en esta materia, por ejemplo "en política de competencia y uso de las TIC para promover la transparencia y la calidad regulatorias" $(2014,42)$.

Particularmente se debe destacar el documento conpes 3816 de 2014 ya mencionado, que sentó las bases para construir una política de mejora normativa en el país. Este documento "busca avanzar en la adopción y adaptación de herramientas, el desarrollo de la institucionalidad, y la generación de capacidades a partir de las cuales sea posible asegurar la calidad de las normas que expide la Rama Ejecutiva del orden nacional" (DNP, 2014,3). En concreto, el objetivo del documento es "sentar las bases para institucionalizar el análisis de impacto normativo (AIN) en la etapa temprana del proceso de emisión de las normas desde la Rama Ejecutiva, como herramienta dirigida a fortalecer la confianza, efectividad y transparencia de la normatividad, en el mediano y largo plazo" (ibíd., 4).

Respecto de este último avance que, además, se encuentra alineado con el interés de que Colombia ingrese a la ocDE, es prematuro hacer un balance de sus resultados. Una lectura del documento permite evidenciar un proceso de ordenación de los instrumentos, instituciones y organizaciones, tanto de los existentes como de los que se introducen por primera vez en el entorno de nacional. Un ejemplo de estos últimos es el análisis de impacto normativo, un instrumento de evaluación ex ante y definición de alternativas a la regulación.

Ahora bien, la revisión del mismo documento también pone en evidencia un problema de formulación de la política. Esto por cuanto se advierte que sin un criterio consistente se decidió dividir en dos documentos de política el desarrollo de una estrategia que debería ser integral para garantizar una regulación eficiente. Así, se observa la generación de dos pilares para la política de mejora regulatoria, cuando los estándares internacionales identifican esta política como un solo pilar, tal y como se señaló al inicio de esta sección.

\footnotetext{
${ }^{9}$ La doble denominación de la política se presenta por la divergencia del concepto "regulación" en el caso colombiano. Así, desde una aproximación jurídica este concepto se reduce a una serie de facultades asociadas a entidades con facultades expresamente regulatorias, como es el caso de las comisiones de regulación creadas con la Ley 142 de 1994. Por otra parte, desde una aproximación económica, el concepto se asocia con cualquier tipo de acción del Estado en la economía y en la sociedad a través de un vehículo normativo.
} 
Retomando la reflexión acerca de las motivaciones de la regulación es posible afirmar, en línea con Rubio, que "las instituciones, en síntesis, afectan las posibilidades de crecimiento. Esas mismas instituciones son las que, en últimas, determinan los costos de transacción" $(1996,67)$.

En el marco de lo anterior, es evidente que las acciones emprendidas en el país en los últimos años buscan configurar un ambiente institucional más proclive a la competitividad. Sin embargo, como se observa en las gráficas anteriores, la posición de Colombia respecto de algunos indicadores relacionados con la calidad de la regulación es bastante discreta.

Así mismo, a pesar de que en Doing business 2015 Colombia se considera el país más reformador, con un mejor ambiente para los negocios, otros indicadores, como el de transparencia en la elaboración de política pública (gráfica 3), lo sitúan en el lugar 84 de 144 y en el 91 en el de eficiencia de los sistemas de solución de controversias (gráfica 4). De hecho, como se presenta en la gráfica 5, Colombia obtiene una calificación de 2,8 sobre 7 , siendo 1 la peor calificación posible, respecto al costo que la regulación impone al sector privado. Esto último quiere decir, de manera preocupante, que la regulación se considera como un sobrecosto.

Gráfica 6

Cumplimiento de la regulación

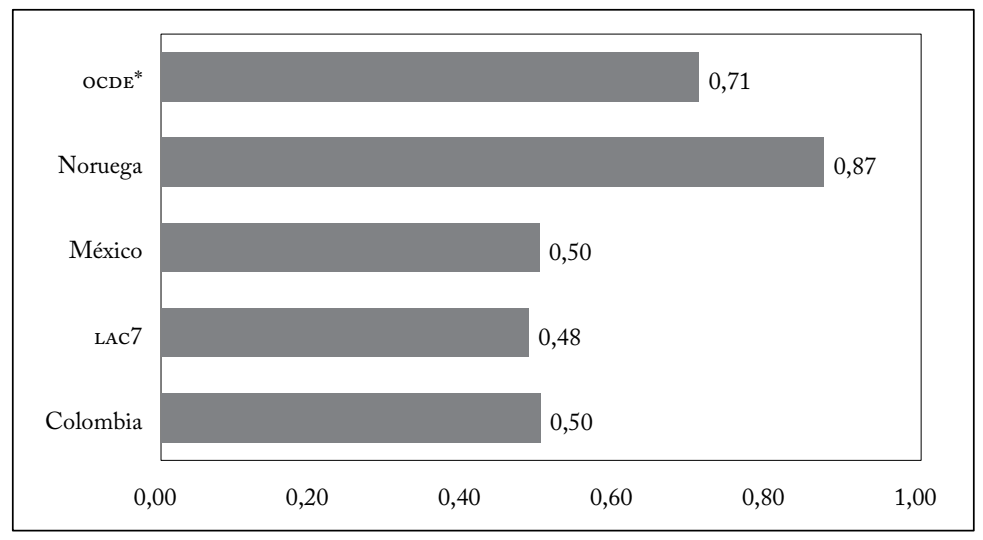

*Promedio simple de los países de la ocDE. La escala va de 0 a 1, donde 1 es la mejor puntuación. Fuente: World Justice Project (2014), 99 economías, Índice de “Estado de derecho”.

Con base en los resultados anteriores se puede afirmar que en $\mathrm{Co}^{-}$ lombia la regulación se considera como un costo de transacción. Lo cual se corrobora al examinar su cumplimiento. Como muestra la 
gráfica 6, al comparar la situación de Colombia con el promedio de LAC7, apenas se obtienen 0,2 puntos por encima. Pero también con respecto a la peor práctica de la ocDE, representada por México con una puntuación idéntica, y la mejor práctica de esta organización, representada por Noruega con 0,87 puntos.

Recurriendo de nuevo a los aspectos presentados en este análisis, se puede decir que en Colombia la intervención del Estado a través de instituciones formales (regulaciones), más que consolidar un ambiente competitivo, es un costo de transacción que desincentiva su cumplimiento. Así, la acción del Estado pierde legitimidad porque la regulación no contribuye a eliminar costos de transacción ex ante ni ex post. Esta situación crea una especie de círculo vicioso en la medida en que la legitimidad del Estado se ve socavada al construir nuevas instituciones que los agentes menosprecian y se carecen de incentivos suficientes para su cumplimiento; lo cual perjudica la legitimidad y la credibilidad del Estado.

Es entonces imprescindible el rápido avance de una mejora regulatoria integral que, estableciendo estándares de calidad de la producción normativa y herramientas de evaluación ex ante como el AIN, restablezca la confianza de los regulados. Sería deseable que el análisis de eficiencia que adelante el AIN tenga en cuenta al menos tres dimensiones. La primera corresponde a los aspectos económicos del proceso de toma de decisiones, buscando "la viabilidad de la decisión, su menor costo posible y el balance entre los costos estimados y los beneficios que se espera alcanzar con la decisión" (Ponce, 2011, 463). La segunda consiste en analizar cómo se materializa la decisión, es decir, el costo de construir un sistema normativo que permita ejecutar la decisión de modo que el costo de elaborarlo no exceda el costo del problema que busca regular. Por último, la evaluación de eficiencia y eficacia, donde se debe analizar si la norma cumple el objetivo para el cual fue elaborada al menor costo posible.

Cada una de estas tres dimensiones se puede interpretar como una posible fuente de vulneración o creación de talanqueras para cumplir los objetivos del Estado social de derecho, como la protección de los derechos fundamentales. Como sugiere Ponce, algunas de las técnicas que se pueden emplear para estudiar la eficiencia, y sobre las cuales hay bastantes reservas, son:

a. Estudio de viabilidad económica de la decisión administrativa. En este caso hay que responder si se cuenta con los recursos necesarios para desarrollar la actividad teniendo en cuenta sus costos. 
b. Menor costo posible. A este respecto el punto central de evaluación es comparar la iniciativa presentada con otras similares. Y evaluar el costo de oportunidad.

c. Análisis costo-beneficio. En este punto se deben calcular los valores esperados de los costos y beneficios de la posible implementación de la decisión.

No obstante, cabe advertir que así se adopten estos métodos de evaluación u otros disponibles para apoyar la construcción de alternativas que maximicen el uso de los recursos ${ }^{10}$, esto no implica que se puedan violar derechos fundamentales. Es entonces esencial emplear mecanismos de ponderación de derechos que, además de contribuir a la legitimidad jurídica y administrativa, eviten que la eficiencia y la eficacia sean los únicos criterios que orienten la acción del Estado. Como se ha señalado en diversas ocasiones, la utilidad de estos dos principios no implica que sean los únicos que habiliten la actuación del Estado. No se pueden dejar de lado otros criterios de igual importancia, como la justicia, la igualdad, la libertad de las personas o el libre desarrollo de la personalidad (Ponce, 2003).

La creación de instituciones no solo abarca fenómenos relacionados con el comportamiento de los agentes y de las organizaciones o la dinámica de los mercados, también incluye la creación de arreglos institucionales que hagan posible controlar la aparición de prácticas oportunistas (Rubio, 1996,2), como las que se derivan de la inexistencia de un mercado perfecto sin plena información, cual suele suceder en los esquemas de supervisión, vigilancia y control desarrollados por las superintendencias.

\section{REFLEXIONES FINALES}

Como plantea Buitrago, "La regulación es la generación de reglas o instituciones cuyo éxito radica en que los incentivos diseñados para actuar sobre las organizaciones públicas y privadas logren orientar su conducta hacia las finalidades del Estado [...] en Colombia no basta hacer bien la regulación, es necesario tener capacidad de adaptación institucional" (2012). Se debe mencionar además que "la posibilidad de que existan mercados eficientes, con bajos costos de transacción, depende entonces de manera fundamental de unas reglas del juego legítimas, creíbles y aceptadas, que permitan definir adecuadamente los derechos que se transfieren en un intercambio y hacer cumplir los contratos que se derivan del mismo" (Rubio, 1996, 3).

${ }_{10}$ Como el cálculo de valores esperados. Para más detalles, ver Monroy y Pinzón (2012). 
Para terminar se pueden sintetizar las enseñanzas o advertencias de la NEI sobre la actividad reguladora:

1. Gran parte del éxito en la construcción de marcos institucionales eficientes depende de la estructura institucional informal. Si no se coordinan las instituciones formales y las informales es muy posible que no se logren los objetivos que busca alcanzar la estructura formal. De modo que es necesario crear esquemas de incentivos que desalienten o impidan la aparición de comportamientos oportunistas o indeseables que afecten indicadores del Estado de derecho y la calidad de la regulación en cuanto al cumplimiento de las obligaciones contractuales y el respeto de los derechos de propiedad.

2. Contar con un sistema jurídico eficiente que permita resolver conflictos y garantice el cumplimiento de la ley. En 1996 se señaló: "Se necesitan menos normas y mejor cumplimiento y respeto de las que queden. Parece indispensable desacelerar, reducir y aún bajar el actual 'stock' legislativo y regulatorio y simultáneamente, fortalecer la justicia” (ibíd., 69). Hoy sigue vigente esa conclusión, como muestra la baja calificación obtenida en el estudio del нsвс (2011) que otorga a Colombia un puntaje de 0,33 en el rubro "Estado de derecho", 0,37 puntos por debajo de la media de cumplimiento mundial en los indicadores de gobernabilidad del Banco Mundial.

3. Construir reglas de juego claras y sencillas que reduzcan los costos de búsqueda y monitoreo de los agentes. A esta advertencia se suma la necesidad de establecer competencias claras a la actividad regulatoria, para que su asignación no sea difusa, las organizaciones públicas y privadas no incurran en costos de búsqueda y contribuya a la legitimidad de la acción. La proliferación de entidades reguladoras aumenta los costos de identificación y negociación cuando una actividad requiere la participación de dos o más organismos con competencias reguladoras, así como los costos de vigilancia del cumplimiento de las diferentes reglas. A este respecto, se deben promover esquemas de vigilancia de carácter integral, es decir, que conciban la vigilancia no de acuerdo con competencias específicas sino con respecto a actores o sectores.

Un marco institucional claro mejora el indicador de calidad del Estado asociado al bajo número de leyes,y más aún si están claramente redactadas, son completas en el tema que abordan y no se contradicen con otras ni a sí mismas (Tanzi, 2000). Un sistema confuso o poco claro eleva los costos de transacción y genera incertidumbre acerca de la capacidad para hacer cumplir la legislación. 
La calidad del sector público mejora cuando las leyes están bien redactadas y cubren todos los aspectos necesarios, cuando no se prestan para interpretaciones encontradas por parte del público o de los funcionarios gubernamentales, cuando no son más numerosas que lo estrictamente necesario y cuando no se contradicen unas con otras. En el pasado varios países -Francia, Italia, Nueva Zelandia y Rusia- han intentado o codificar las leyes existentes o simplificarlas. Sin embargo, estas iniciativas, que solo rendirían beneficios a largo plazo, no suelen recibir gran apoyo político. Por ello, estos intentos se abandonan frecuentemente antes de que rindan los frutos esperados (ibíd., 15).

4. Un punto crítico al que aporta notablemente el enfoque de la NEI es el de la reglamentación.

Un sector público de alta calidad debe tener reglas claras suficientes para orientar la actividad económica (y otras), pero no tantas ni tan vagas que otorguen poder desmedido a los burócratas y creen confusión entre quienes toman las decisiones económicas. En general, las reglas deben especificar lo que no se permite más que autorizar lo que se permite. En lo posible el ejercicio de la discreción por parte de los burócratas debe mantenerse en un mínimo (ibíd., 14).

\section{REFERENCIAS BIBLIOGRÁFICAS}

1. Arias P., A. "El neoinstitucionalismo y sus aportes a la teoría de la organización”, Gestión y Región 6, 2008, pp. 31-63.

2. Ariño O., G. Principios de derecho público económico, Bogotá, Universidad Externado de Colombia, 2003.

3. Ariño O., G. y J. M. de la Cuétara. "Algunas ideas básicas sobre la regulación de sectores estratégicos”, Cuadernos de Derecho Público 9, 2000, pp. 9-26.

4. Ayala E., J. Instituciones y economia: una introducción al neoinstitucionalismo económico, México DF, Fondo de Cultura Económica, 2005.

5. Baldwin, R.; M. Cave y M. Lodge. Understanding regulation: Theory, strategy, and practice, Nueva York, Oxford University Press, 2012.

6. Banco Mundial. Doing business 2012: haciendo negocios en un mundo más transparente, Washington DC, Banco Mundial, 2011.

7. Banco Mundial. Interactive data access: The worldwide governance indicators, 2014, [http://info.worldbank.org/governance/wgi/index. aspx\#reports].

8. Banco Mundial. Reports: Doing Business, Washington Dc, 2014, [http://espanol.doingbusiness.org/reports/global-reports/doingbusiness-2015].

9. Barcelona L., J. “Aproximación jurídica a los fines, principios y técnicas de la regulación económica", M. Arenillas, ed., La administración pública entre dos siglos, Madrid, INAP, 2010, pp. 1299-1317.

10. Boyer, R. La teoría de la regulación: un análisis crítico, Buenos Aires, Humanitas, 1989.

11. Buitrago, R. J. Conferencia Reforma del Estado-Modelo empresarial del Estado, Bogotá, Universidad Externado de Colombia, 2012.

12. Carro F., J. L. y R. Gómez-F. "La protesta reglamentaria del gobierno y la Constitución", 34 artículos seleccionados de la Revista de 
Administración Pública con ocasión de su centenario, Madrid, INAP, 1983, pp. 943-986.

13. Cepeda E., M. J. Sentencia C-779, Bogotá, Corte Constitucional, 2003.

14. Chang, H.-J. "La relación entre instituciones y el desarrollo económico. Problemas teóricos claves”, Revista de Economía Institucional 8, 14, 2006, pp. 125-136.

15. Coase, R. H. “The nature of the firm”, Economica 4, 16, 1937, pp. 386-405.

16. Coase, R. H. "The problem of social cost", The Journal of Law and Economics 3, 1960, pp. 1-44.

17. Coase, R. H. La empresa, el mercado y la ley, Madrid, Alianza, 1994.

18. Cooter, R. y T. Ulen. Derecho y economía, México DF, Fondo de Cultura Económica, 1997.

19. Correa H., M. Libertad de empresa en el Estado social de derecho, Bogotá, Universidad Externado de Colombia, 2009.

20. Cubero M., J. I. "Regulación, iniciativa pública económica y libre competencia: hacia un modelo sin inmunidades", Revista de Administración Pública 184, 2011, pp. 121-156.

21. Cuevas, H. “Teoría económica del Estado”, H. Cuevas, ed., Teorías jurídicas y económicas del Estado, Bogotá, Universidad Externado de Colombia, 2002, pp. 67-169.

22. Custos, D. "La noción estadounidense de regulación”, G. Marcou y M. Franck, eds., Derecho de la regulación, los servicios públicos y la integración regional, Bogotá, Universidad del Rosario, 2009, pp. 190-218.

23. Departamento Administrativo de la Presidencia de la República. Decreto 1345, Bogotá, DAPRE, 2010.

24. Departamento Nacional de Planeación. “conpes 3816. Mejora normativa: análisis de impacto”, Bogotá, DNP, 2014.

25. Djankov, S.; C. McLiesh y R. M. Ramalho. "Regulation and growth", Economics Letters 92, 3, 2006, pp. 395-401.

26. Foro Económico Mundial. Reporte Global de Competitividad 20142015, Ginebra, FEM, 2014.

27. Gorgens, T.; M. Paldam y A. Wuertz. "How does public regulation affect growth?”, University of Aarhus, working paper 2003-14, 2003.

28. Grau, E. R. "La regulación en Brasil”, G. Marcou y F. Moderne, eds., Derecho de la regulación, los servicios públicos y la integración regional, Bogotá, Universidad del Rosario, 2009, pp. 219-251.

29. Guasch, J. L. y P. Spiller. "The cost and benefits of regulation: The call for regulatory reform”, J. L. Guasch y P. Spiller, eds., Managing the regulatory process: Design, concepts, issues, and the Latin America and Caribbean Story, Washington DC, World Bank, 1999, pp. 1-30.

30. Holmes, S. y C. R. Sunstein. El costo de los derechos: por qué la libertad depende de los impuestos, Buenos Aires, Siglo xxi, 2011.

3 I. Hsвс. The world in 2050. Quantifying the shift in the global economy, Londres, нsвс Global Research, 2011.

32. Ibáñez N., J. E. "Alcance y límites de las potestades reguladora y reglamentaria”, Vniversitas 106, 2003, pp. 10-93. 
33. Jacobzone, S.; F. Steiner et al. "Assessing the impact of regulatory management systems: Preliminary statistical and econometric estimate", OECD working papers on Public Governance 17, 2010.

34. Jalilian, H.; C. Kirkpatrick y D. Parker. "The impact of regulation in developing countries: A cross sectional analysis", World Development 35, 1, 2007, pp. 87-103.

35. Laufer, R. y A. Burlaud. "Crisis de legitimidad en las grandes organizaciones”, Dirección pública: gestión y legitimidad, Madrid, InAP, 1989, pp. 25-56.

36. Mankiw, N. G. Principios de economia, Madrid, McGraw Hill, 1998.

37. Monroy C., D. y C. M. Pinzón. "Análisis económico de los derechos colectivos y su mecanismo de protección jurisdiccional en Colombia: el papel de los incentivos, la acción colectiva y la provisión de bienes públicos”, Con-Texto 36, 2012, pp. 11-58.

38. Muñoz M., S. "Hacia un nuevo derecho administrativo", A. Blasco, ed., El derecho público de la crisis económica. Transparencia y sector público, Palma de Mallorca, inap, 2011, pp. 191-237.

39. Nelson, R. R. y B. N. Sampat. "Las instituciones como factor que regula el desempeño económico", Revista de Economía Institucional 5, 2001, pp. 17-51.

40. North, D. Economic performance through time, Estocolmo, Prize in Economic Sciences, Nobel Prize, 1993.

41. North, D. Instituciones, cambio institucional y desempeño económico, México DF, Fondo de Cultura Económica, 2006.

42. ocDE. Estudio de la OCDE sobre la politica regulatoria en Colombia: más allá de la simplificación administrativa, París, ocde, 2014.

43. ocDE. Recomendación del Consejo sobre politica y gobernanza regulatoria, París, OCDE, 2012.

44. ocDE. Regulatory policy and governance: Supporting economic growth and serving the public interest, París, OCDE, 2011.

45. Parker, D. y C. Kirkpatrick. The economic impact of regulatory policy: A literature review of quantitative evidence, París, OCDE, 2012.

46. Pinzón C., M. A. Aproximaciones al análisis económico del derecho, Bogotá, Universidad Externado de Colombia, 2010.

47. Pinzón C., M. A. "La nueva economía institucional: un puente para el estudio de las relaciones entre derecho y desarrollo", C. A. Naranjo et al., El derecho del consumo, Bogotá, Universidad Externado de Colombia, 2013, pp. 169-225.

48. Ponce S., J. "La calidad en el desarrollo de la discrecionalidad reglamentaria: teorías sobre la regulación y la adopción de buenas decisiones normativas por los gobiernos y las administraciones", Revista de Administración Pública 162, 2003, pp. 89-144.

49. Ponce S., J. "Crisis económica, deberes jurídicos de economía y eficiencia y derecho administrativo", A. Blasco, ed., El derecho público en la crisis económica. Transparencia y sector público. Hacia un nuevo derecho administrativo, Palma de Mallorca, INAP, 2011, pp. 459-473.

50. Prats C., J. "Derecho y management en las administraciones públicas", Q. Brugué y J. Subirats, eds., Lecturas de gestión pública, Madrid, Ministerio de Administraciones Públicas, 1996, pp. 181-198. 
51. Predieri, A. "Estado y mercado: La ósmosis como elemento cualificador y legitimante", Revista del Centro de Estudios Constitucionales 17, 1994, pp. 9-68.

52. Radaelli, C. y F. de Francesco. Better regulation for growth: Governance frameworks and tools for effective regulatory reform, regulatory quality indicators, Washington DC, World Bank, 2010.

53. Ramírez H., F. La politica de competencia y el proceso de regulación en México, 1993-1999, México DF, Universidad Autónoma de México, 2001.

54. Roth D., A.-N. Politicas públicas: formulación, implementación y evaluación, Bogotá, Aurora, 2002.

55. Rubio, M. Reglas de juego y costos de transacción en Colombia, Bogotá, CEDE, 1996.

56. San Emeterio, N. Nueva economía institucional, Madrid, Síntesis, 2006.

57. Sappington, D. E. “Designing incentive regulation”, Review of Industrial Organization 9, 3, 1994, pp. 245-272.

58. Smith, A. Investigación sobre la naturaleza y causas de la riqueza de las naciones, México DF, Fondo de Cultura Económica, 1994.

59. Stiglitz, J. E. La economía del sector público, Barcelona, Antoni Bosch, 2000.

60. Tanzi, V. "El papel del Estado y la calidad del sector público”, Revista de la Cepal 71, 2000, pp. 7-22.

61. Williamson, O. E. Las instituciones económicas del capitalismo, México DF, Fondo de Cultura Económica, 2009.

62. World Bank. Worldwide Governance Indicators, Washington Dc, World Bank, 2011, [http://info.worldbank.org/governance/wgi/index.asp].

63. World Economic Forum. The global competitiveness Report 2011-2012, Ginebra, weF, 2011.

64. World Justice Project. Rule of Law Index 2014, Washington, wJP, 2014. 